Editorial:

\title{
COVID-19 Pandemic and Neurological Presentations: Body of Evidence
}

\author{
Payman Vahedi ${ }^{1,2}$ (1) \\ 1. Assistant Professor of Neurosurgery, Department of Neurosurgery, School of Medicine, Tehran Medical Sciences Branch, Islamic Azad University of Medical Sci- \\ ences, Tehran, Iran \\ 2. Neurosurgeon and Spine Consultant, Department of Neurosurgery, Milad Hospital, Tehran, Iran
}

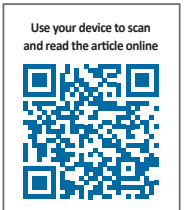

CFtation: Payman V. COVID-19 Pandemic and Neurological Presentations: Body of Evidence. Iran J Neurosurg. 2020; 6(1):1-2 http://dx.doi.org/10.32598/irjns.6.1.1

dol: $:$ http://dx.doi.org/10.32598/irjns.6.1.1

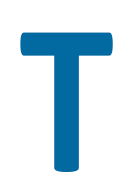

he rapid emergence of COVID-19 pandemic by SARS-Cov2 at the beginning of the year 2020 has caused a state of confusion worldwide. As the virus exhibits some unknown manifestations barely attributable to a lone respiratory infection, the medical workforce is challenged with a big battle against an infectious organism since the influenza pandemic in the 1920s. According to the latest report of The Center for Disease Control (CDC), affected countries now count up to more than 180 [1]. The whole globe is struggling with limited medical resources and facilities to treat the exponentially increasing number of patients who may ultimately need prolonged ICU care and interdisciplinary management.

It is now evident that COVID-19is not a sole respiratory infection and has the tendency to become a multi-organ infection. It is believed that this extraordinary feature may be due to the potential of the virus to attack Angiotensin-Converting Enzyme II (ACE II) receptors on the endothelial walls of different tissues and organs leading to localized vasculitis [2].

The central nervous system involvement was first recognized by hyposmia/anosmia and hypogeusia and the theory of direct CNS invasion by the virus through the cribriform plate of the ethmoid bone and olfactory epithelium was popularized [3]. Further, the hematog- enous spread of the virus was hypothesized by some authors [4]. Although different in nature, these two current possible mechanisms for CNS involvement agree on the potential SARS-COv-2 neurotropism, which may explain a wide spectrum of neurological manifestations occurring during the infection. These include severe headache, altered level of consciousness, encephalitis, Ischemic Cerebrovascular Accidents (ICVA), seizures, and intracerebral hemorrhagic infarcts. Of note, it is also hypothesized that fatal respiratory syndrome may be subsequent to the involvement of brainstem nuclei and should be considered as a consequence of CNS involvement [4].

Although the literature is being completed on the neurologic manifestations of COVID-19 with tremendous interest, most of the studies are currently of a low level of evidence and are confined to simple case reports or retrospective series. Accordingly, it is difficult to relate the occurrence of such a presentation to COVID-19. There is yet no sufficient data in the literature whether the neurological manifestations are primarily caused by the virus or are the results of secondary CNS involvement. This may be especially true in a subgroup of patients admitted with frail health conditions like elderly people or those with uncontrolled systemic diseases like hypertension, diabetes mellitus or hyperlipidemia who may experience secondary neurologic complica-

* Corresponding Author:

Payman Vahedi, MD.

Address: Department of Neurosurgery, School of Medicine, Tehran Medical Sciences Branch, Islamic Azad University of Medical Sciences, Tehran, Iran Tel: +98 (21) 33348036

E-mail: payman.vahedi@gmail.com 
tions after hospitalization and as a result of the severe burden of the associated respiratory infection leading to hypoxia. Logically, attributing some presentations, e.g. ICVA to COVID-19 could only be verified by autopsy findings; while a positive result for reverse Transcription Polymerase Chain Reaction (RT-PCR) for SARS-COV-2 in CSF may provide enough clues in patients diagnosed with COVID-19 meningitis or encephalitis.

At present, it is highly recommended that the global neurological and neurosurgical community should remain alert on the possible primary or secondary CNS involvement by COVID-19. Hence, until being proved otherwise, it is advisable that a high level of suspicion should be sought in patients presenting with neurological manifestations who have had a history of recent respiratory symptoms. This also applies to any urgent neurosurgical intervention on patients appearing with life-threatening emergencies.

\section{Ethical Considerations}

Compliance with ethical guidelines

No animal or human research reported in this letter, there was no need for ethics board approval.

Funding

This research did not receive any specific grant from funding agencies in the public, commercial, or not-forprofit sectors.

\section{Conflict of interest}

The author declared no conflict of interest.

\section{References}

[1] Centers for Disease Control and Prevention. Coronavirus (COVID-19) [Internet]. 2020 [Updated 2020 Jul 28]. Available from: https://www.cdc.gov/coronavirus/2019-ncov/global-covid-19/ world-map.html

[2] Li Y, Zhou W, Yang L, You R. Physiological and pathological regulation of ACE2, the SARS-CoV-2 receptor. Pharmacological Research. 2020; 157:104833. [DOI:10.1016/j.phrs.2020.104833] [PMID] [PMO CID]

[3] Mao L, Jin H, Wang M, Hu Y, Chen S, He Q, et al. Neurologic manifestations of hospitalized patients with Coronavirus Disease 2019 in Wuhan, China. JAMA Neurology. 2020; 77(6):683-90. [DOI:10.1001/ jamaneurol.2020.1127] [PMID] [PMCID]

[4] Baig AM. Neurological manifestations in COVID-19 caused by SARS-CoV-2. CNS Neuroscience \& Therapeutics. 2020; 26(5):499501. [DOI:10.1111/cns.13372] [PMID] [PMCID] 